\title{
Leaflet thickening and stent geometry in sutureless bioprosthetic aortic valves
}

\author{
Raquel Themudo $^{1,2} \cdot$ Mikael Kastengren $^{3,4} \cdot$ Elin Bacsovics Brolin ${ }^{5,6} \cdot$ Kerstin Cederlund $^{5} \cdot$ Anders Svensson $^{1,5}$. \\ Magnus Dalén ${ }^{4,7}$
}

Received: 14 July 2019 / Accepted: 10 January 2020 / Published online: 16 January 2020

(c) The Author(s) 2020

\begin{abstract}
Underexpansion of transcatheter heart valves and the surgically implanted Perceval sutureless aortic valve bioprosthesis has been suggested as an underlying mechanism for hypo-attenuated leaflet thickening (HALT). This was a single-center prospective observational study that included 47 patients who underwent surgical aortic valve replacement with the Perceval sutureless bioprosthesis (LivaNova, London, United Kingdom) from 2012 to 2016 and were studied by four-dimensional cardiac computed tomography (CT). The association between overall and regional expansion and the prevalence of HALT was analyzed. In total 46 patients were included in the analysis. HALT was found in $39.1 \%$ of patients and the mean overall prosthesis expansion was 75.5 $\pm 5.2 \%$ (range 64.6-84.8\%). Overall expansion did not differ between patients with HALT compared with patients without HALT (mean overall expansion $74.0 \pm 5.2 \%$ vs. $76.5 \pm 5.0 \%, P=0.11$ ). The prevalence of HALT was lower in patients with overall expansion $>80 \%$ compared to patients with expansion $<80 \%$ expansion though not significantly ( $20 \%$ vs. $44.4 \%, P=0.16$ ). None or trivial regional underexpansion was found in $94.7 \%$ of coronary cusps. There was no significant association between regional underexpansion and the prevalence of HALT (mean coronary cusp angle $120 \pm 8^{\circ}$ vs. $119 \pm 10^{\circ}, P=0.53$ ). The prevalence of HALT and overall underexpansion was high in the Perceval sutureless bioprosthetic valve. Overall underexpansion was not associated with HALT. Whether severe overall underexpansion increases the risk for HALT requires further study. Regional underexpansion was uncommon in the Perceval sutureless bioprosthetic valve and not associated with HALT.
\end{abstract}

Clinical trial registration Unique identifier: NCT03753126 (http://www.clinicaltrials.gov).

Keywords Aortic valve surgery $\cdot$ Bioprosthesis $\cdot$ Four-dimensional cardiac computed tomography $\cdot$ Leaflet thickening · Stent geometry

Raquel Themudo and Mikael Kastengren have contributed equally to this work.

Mikael Kastengren

mikael.kastengren@sll.se

1 Department of Radiology, Karolinska University Hospital, Stockholm, Sweden

2 Department of Clinical Physiology, Karolinska Institutet, Stockholm, Sweden

3 Department of Cardiology, Karolinska University Hospital, C11:28, 17176 Stockholm, Sweden

\section{Introduction}

Hypo-attenuated leaflet thickening (HALT), with or without associated reduced leaflet motion, is prevalent in both transcatheter and surgically implanted bioprosthetic aortic valves [1-3]. The underlying cause has been speculated to be thrombogenic as anticoagulation therapy with warfarin

4 Department of Molecular Medicine and Surgery, Karolinska Institutet, Stockholm, Sweden

5 Department of Clinical Science, Intervention and Technology, Karolinska Institutet, Stockholm, Sweden

6 Department of Radiology, Capio S:t Göran Hospital, Stockholm, Sweden

7 Department of Cardiac Surgery, Karolinska University Hospital, Stockholm, Sweden 
has been associated with restored cusp thickness in patients with HALT [2]. The prevalence of HALT has been associated with cardiovascular events $[2,4]$.

Recently, bioprosthetic valve underexpansion has been hypothesized to affect leaflet thickening in aortic bioprosthetic valves [5]. In transcatheter valves, regional underexpansion of transcatheter aortic valve cusps has been reported to be associated with an increased prevalence of HALT [5]. In the surgically implanted Perceval sutureless aortic valve bioprosthesis, overall underexpansion of the valve and failure to achieve circularity has been speculated to cause HALT $[6,7]$.

The aim of this study was to investigate the potential relationship between four-dimensional (4D) cardiac computed tomography (CT)-derived overall and regional underexpansion and HALT in the Perceval sutureless bioprosthetic valve.

\section{Methods}

\section{Study design}

This was a single-center prospective observational study approved by the regional Human Research Ethics Committee, Stockholm, Sweden. Informed consent was obtained from patients meeting the inclusion criteria.

\section{Study group}

All patients who had undergone surgical aortic valve replacement (AVR) with implantation of the Perceval sutureless bioprosthesis (LivaNova, London, United Kingdom) at Karolinska University Hospital in Stockholm, Sweden (October 2012 to February 2016) were eligible. The criterion to implant the Perceval sutureless bioprosthesis was aortic stenosis with indication for primary isolated nonemergent AVR. Implantation was considered feasible if the ratio between the diameter of the sinotubular junction and the diameter of the aortic annulus did not exceed 1.3. A type 0 bicuspid aortic valve was a contraindication for Perceval sutureless bioprosthesis implantation. Exclusion criteria were severely impaired renal function (glomerular filtration

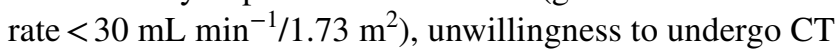
examination, or inability to participate in the examination for logistical reasons.

\section{Sutureless surgical aortic valve replacement}

Patients underwent sutureless bioprosthesis AVR via either full sternotomy or partial upper hemisternotomy. Cardiopulmonary bypass was established with central arterial and central or peripheral percutaneous venous cannulation. Implantation was performed as previously described [8].

\section{Antithrombotic regime}

According to the standard antithrombotic protocol for aortic bioprostheses at our center, postoperative antithrombotic treatment consisted of low molecular weight heparin until full mobilization and life-long treatment with acetylsalicylic acid $75 \mathrm{mg}$ once daily. Patients without a preoperative indication for oral anticoagulation did not receive oral anticoagulants postoperatively. In patients preoperatively treated with long-term oral anticoagulation, this treatment was paused 3 days prior to the operation without bridging with low molecular weight heparin. In these patients, anticoagulation therapy (without additional acetylsalicylic acid) was re-administered at day 1 postoperatively.

\section{Cardiac computed tomography data acquisition}

Oral metaprolol (50-100 $\mathrm{mg}$ according to heart rate) was administered $1 \mathrm{~h}$ prior to the $\mathrm{CT}$ examination. All patients were scanned using a dual-source $2 \times 64$ row multidetector computed tomograph (Siemens Somatom Definition Flash; Siemens Healthcare, Forchheim, Germany). Scanning was performed with retrospective ECG scanning, 100-120 kVp depending on patient's weight, automatic dose modulation (CARE dose) and full-dose R-R scanning (no ECG $\mathrm{mA}$ modulation), $64 \times 0.6 \mathrm{~mm}$ detector collimation, $0.28 \mathrm{~s}$ rotation time ( $75 \mathrm{~ms}$ temporal resolution). An intravenous contrast agent with a concentration of $320 \mathrm{mg}$ iodine per $\mathrm{mL}$ (Visipaque, GE Healthcare, Stockholm, Sweden) was used with a fixed injection time of $8 \mathrm{~s}$ and a dose of $200 \mathrm{mg}$ iodine $/ \mathrm{kg}$ body weight. A contrast dose increase of $20 \%$ was used for patients scanned with $120 \mathrm{kVp}$. Delay time was defined using the test bolus technique. Data were reconstructed to $0.75 \mathrm{~mm}$ slice thickness with an increment of $0.4 \mathrm{~mm}$ at $5 \%$ intervals ( 20 phases) throughout the R-R interval and at best systolic and diastolic phases. A more detailed description of CT data acquisition has previously been described [3]. For in vitro assessment of the Perceval sutureless bioprosthesis in each size, we used the same computed tomograph and scanning parameters as for the in vivo CT examinations.

\section{Cardiac computed tomography analysis}

CT data analysis was performed using the Syngo.via software (Siemens Healthcare) on a PACS workstation. 4D cardiac CT images were analyzed independently by two experienced readers (level 2 according to American College of Cardiology (ACC) Foundation/American Heart Association (AHA) levels of CT reading competence) [9]. Joint readings 
involving a third experienced reader were subsequently performed to reach a consensus. For assessment of leaflet anatomy, 1-mm-thick multiplanar reformatted reconstruction (MPR) images in the plane of the leaflet and in a perpendicular plane to the leaflet being assessed were used. Still images from selected phases of the cardiac cycle, as well as dynamic images of the entire cardiac cycle, were used. An examination was considered non-diagnostic if artifacts prevented reliable assessment of one or more valve leaflet. HALT was defined according to the definition previously proposed [10]: evidence of one or more leaflet with hypoattenuated thickening identifiable in at least two different MPR projections. HALT is illustrated in Fig. 1.

Bioprosthetic valve expansion was analyzed at the valvular level in the end-diastolic phase of the heart cycle using 1-mm-thick MPR images. Overall bioprosthetic valve geometry was measured with regard to maximal and minimal stent diameters $(\mathrm{mm})$ and cross-sectional area $\left(\mathrm{mm}^{2}\right)$. In vitro measurements were made for each available size (small, medium, large, extra large) of the Perceval sutureless bioprosthesis using CT images to assess maximal prosthesis dimensions. The degree of overall expansion of the implanted prosthesis was calculated as the ratio between the in vivo cross-sectional area (CSA) and the in vitro CSA of the implanted valve according to the formula (in vivo $\mathrm{CSA} /$ in vitro CSA $) \times 100[5,7]$. The degree of eccentricity (\%) was calculated as ([max diameter - min diameter $] / \max$ diameter) $\times 100[5]$.

Regional expansion was measured at the coaptation level in the end-diastolic phase of the heart cycle using 1 -mm-thick MPR images, using the angle $\left(^{\circ}\right.$ ) formed by the border of each prosthetic leaflet and the center point of the valve. This angle was measured for all three prosthetic leaflets (non-coronary cusp [NCC], right coronary cusp [RCC], and left coronary cusp [LCC]). Normal regional valve expansion was defined as an expansion angle $>114^{\circ}\left(120^{\circ}\right.$ with a 5\% error margin). Regional

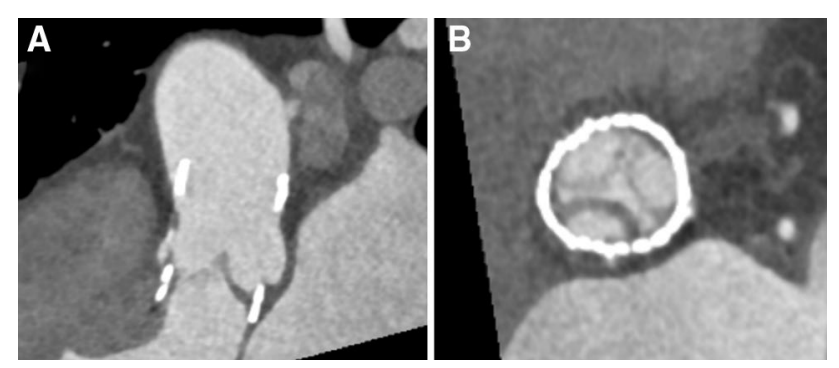

Fig. 1 Cardiac computed tomography multiplanar reformatted reconstructions of a perceval sutureless aortic valve bioprosthesis in middiastole. One cusp was markedly thickened with hypo-attenuated leaflet thickening (panel A). The three valve leaflets are shown simultaneously: two of them normal and one cusp with hypo-attenuated leaflet thickening (panel B) valve underexpansion was defined as trivial if angle $102^{\circ}-114^{\circ}$, mild if angle $90^{\circ}-102^{\circ}$, moderate if angle $78^{\circ}-90^{\circ}$, and severe if angle $<78^{\circ}$ [5]. CT images illustrating how overall expansion, regional expansion, and eccentricity were measured and calculated are presented in Fig. 2.

\section{Study outcomes}

Primary outcomes were overall and regional underexpansion of the bioprosthetic valve. Secondary outcome was eccentricity of the bioprosthetic valve.

\section{Statistical methods}

Variables were described using frequencies and percentages for categorical variables, and means and standard deviations (SD) for continuous variables. Continuous variables were compared using the $t$ test, and categorical or binary variables were compared using Pearson's $\chi^{2}$ test. A two-sided $P$ value of less than 0.05 was considered to indicate statistical significance. Statistical analyses were performed using Stata 15.1 (Stata Corp, College Station, TX, USA).
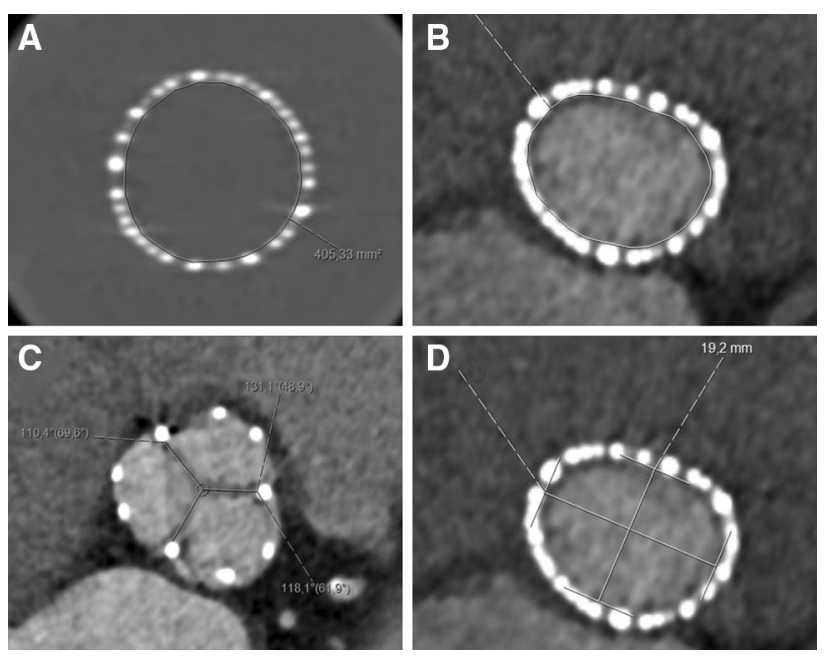

Fig. 2 Cardiac computed tomography analyses. a Measurement of the in vitro cross-sectional area in a Perceval bioprosthetic valve. b Measurement of in vivo cross-sectional area in an implanted bioprosthesis. The degree of overall expansion of the implanted prosthesis was calculated as the ratio between the in vivo cross-sectional area (CSA) and the in vitro CSA of the implanted valve according to the formula (in vivo $\mathrm{CSA} /$ in vitro $\mathrm{CSA}$ ) $\times 100$. $\mathbf{c}$ Regional expansion measured using the angle $\left({ }^{\circ}\right)$ formed by the border of each prosthetic leaflet and the center point of the valve. d The degree of eccentricity (\%) was calculated as ([max diameter-min diameter $] / \max$ diameter $) \times 100$ 


\section{Results}

\section{Study group}

Between October 2012 and February 2016, a total of 58 patients received a Perceval sutureless bioprosthesis. One patient died before initiation of the study at postoperative day 127. Fifty-seven patients were eligible for participation in the study. Ten patients were not included in the study (one with impaired renal function, seven unwilling to participate in the study, two who were indisposed for logistical reasons). 4D cardiac CT was performed between February and March 2016 in 47 patients. All 4D cardiac CT examinations were diagnostic regarding the evaluation of HALT. One examination was non-diagnostic for overall expansion of the bioprosthesis and this patient was excluded. Three examinations were non-diagnostic for regional bioprosthesis expansion due to motion artifacts at the end-diastolic phase of the heart cycle and these patients were excluded. Consequently, a total of 46 patients were included in the analysis of overall underexpansion, and 44 patients were included in the analysis of regional underexpansion. Patient characteristics at the time of surgery are shown in Table 1. 4D cardiac CT was performed at a median of 491 days (quartile 1: 287, quartile 3: 933 days) postoperatively.

\section{Hypo-attenuated leaflet thickening}

HALT was found in 18 (39.1\%) patients, of which 10 (55.6\%) had one affected leaflet, six (33.3\%) had two affected leaflets, and two (11.1\%) had HALT of all three leaflets. As a result, 28/132 leaflets (21.2\%) were found to have HALT (10 RCC, 8 LCC, and 10 NCC).

\section{Overall expansion}

The mean overall prosthesis expansion was $75.5 \pm 5.2 \%$ in the total population. There was no significant association between overall expansion and the prevalence of HALT $(74.0 \pm 5.2 \%$ in patients with HALT vs. $76.5 \pm 5.0 \%$ in patients without HALT, $P=0.11$; Fig. 3 ). As evaluated on the CT examination, ten patients $(21.7 \%)$ reached an overall expansion of $\geq 80 \%$. The prevalence of HALT was lower in patients with overall expansion $>80 \%$ compared to patients with expansion $<80 \%$ expansion though not significantly ( $20 \%$ vs. $44.4 \%, P=0.16$; Fig. 4 ).

\section{Regional underexpansion}

The prevalence of regional underexpansion is presented in Table 2. There was no significant association between regional underexpansion and the prevalence of HALT (Fig. 5, Table 2).
Table 1 Patient characteristics and prosthesis size

\begin{tabular}{lllll}
\hline & $\begin{array}{l}\text { Total popula- } \\
\text { tion }(n=46)\end{array}$ & No HALT $(n=28)$ & HALT $(n=18)$ & $P$ value \\
\hline Age, years, mean \pm SD & $74.5 \pm 5.5$ & $75.7 \pm 4.0$ & $72.8 \pm 7.8$ & 0.092 \\
Female sex & $35(76 \%)$ & $21(75 \%)$ & $14(78 \%)$ & 0.83 \\
Body mass index, kg/m ${ }^{2}$, mean \pm SD & $27.8 \pm 5.0$ & $27.7 \pm 3.8$ & $28.1 \pm 6.5$ & 0.80 \\
Left ventricular ejection fraction $\leq 50 \%$ & $43(93 \%)$ & $26(93 \%)$ & $17(94 \%)$ & 0.83 \\
Estimated glomerular filtration rate, & $67.5 \pm 18.2$ & $68.9 \pm 16.0$ & $65.2 \pm 21.5$ & 0.51 \\
$\quad$ mL min ${ }^{-1} 1.73 \mathrm{~m}^{-2}$, mean $\pm \mathrm{SD}$ & & & & \\
Diabetes mellitus & $10(22 \%)$ & $4(14 \%)$ & $6(33 \%)$ & 0.13 \\
Hypertension & $33(72 \%)$ & $20(71 \%)$ & $13(72 \%)$ & 0.95 \\
Cerebrovascular event & $6(13 \%)$ & $4(14 \%)$ & $2(11 \%)$ & 0.76 \\
Chronic lung disease & $4(9 \%)$ & $4(14 \%)$ & 0 & 0.09 \\
Peripheral artery disease & 0 & 0 & 0 & - \\
Previous myocardial infarction & $1(2 \%)$ & $0(0 \%)$ & $1(6 \%)$ & 0.21 \\
Previous cardiac surgery & $1(2 \%)$ & $1(4 \%)$ & 0 & 0.42 \\
EuroSCORE II, mean \pm SD & $2.03 \pm 1.08$ & $2.10 \pm 1.18$ & $1.91 \pm 0.92$ & 0.57 \\
Prosthesis size & & & & 0.41 \\
Small & $4(9 \%)$ & $4(14 \%)$ & 0 & $7(39 \%)$ \\
Medium & $17(37 \%)$ & $10(36 \%)$ & $9(50 \%)$ & \\
Large & $20(43 \%)$ & $11(39 \%)$ & $3(11 \%)$ & \\
Extra large & $5(11 \%)$ & $3(11 \%)$ & & \\
\hline
\end{tabular}

Data are $n(\%)$ unless otherwise noted

EuroSCORE II European System for Cardiac Operative Risk Evaluation Score II, HALT hypo-attenuated leaflet thickening, $S D$ standard deviation 


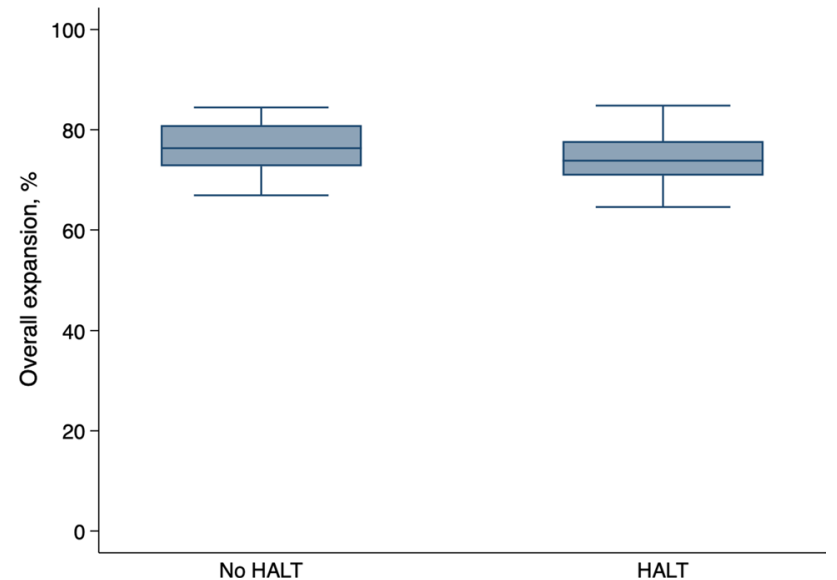

Fig. 3 Overall expansion of the implanted prosthesis in relation to hypo-attenuated leaflet thickening. The whiskers denote the lowest/ highest data points still within 1.5 interquartile range of the lower/ upper quartile

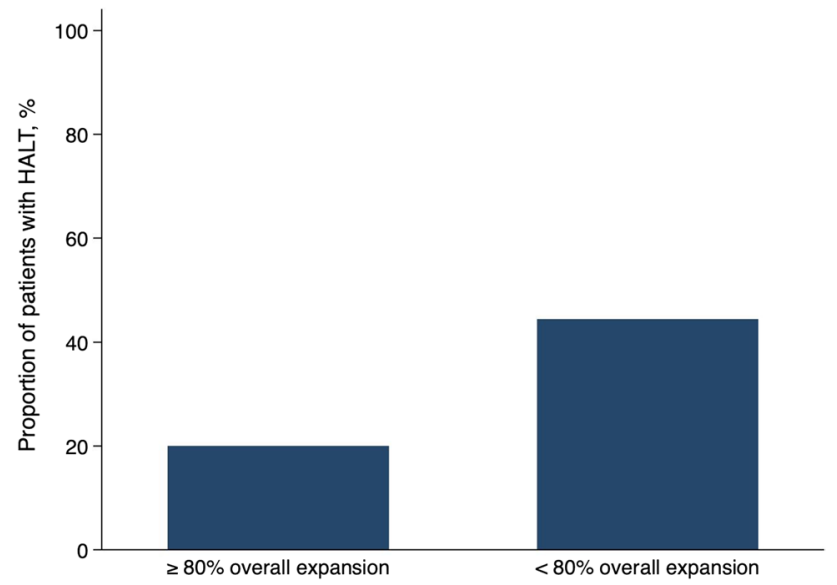

Fig. 4 Proportion of patients with hypo-attenuated leaflet thickening in patients with $<80 \%$ overall expansion versus patients with $\geq 80 \%$ overall expansion of the implanted prosthesis

\section{Eccentricity}

The mean eccentricity index was $16.4 \pm 7.7 \%$ in the total population. There was no significant association between eccentricity index and the prevalence of HALT $(17.0 \pm 8.1 \%$ in patients with HALT vs. $16.0 \pm 7.6 \%$ in patients without HALT, $P=0.66$; Fig. 6). Eccentricity index of more than $15 \%$ was similar in patients with and without HALT $(78 \%$ vs. $61 \%$, $P=0.23$ ).

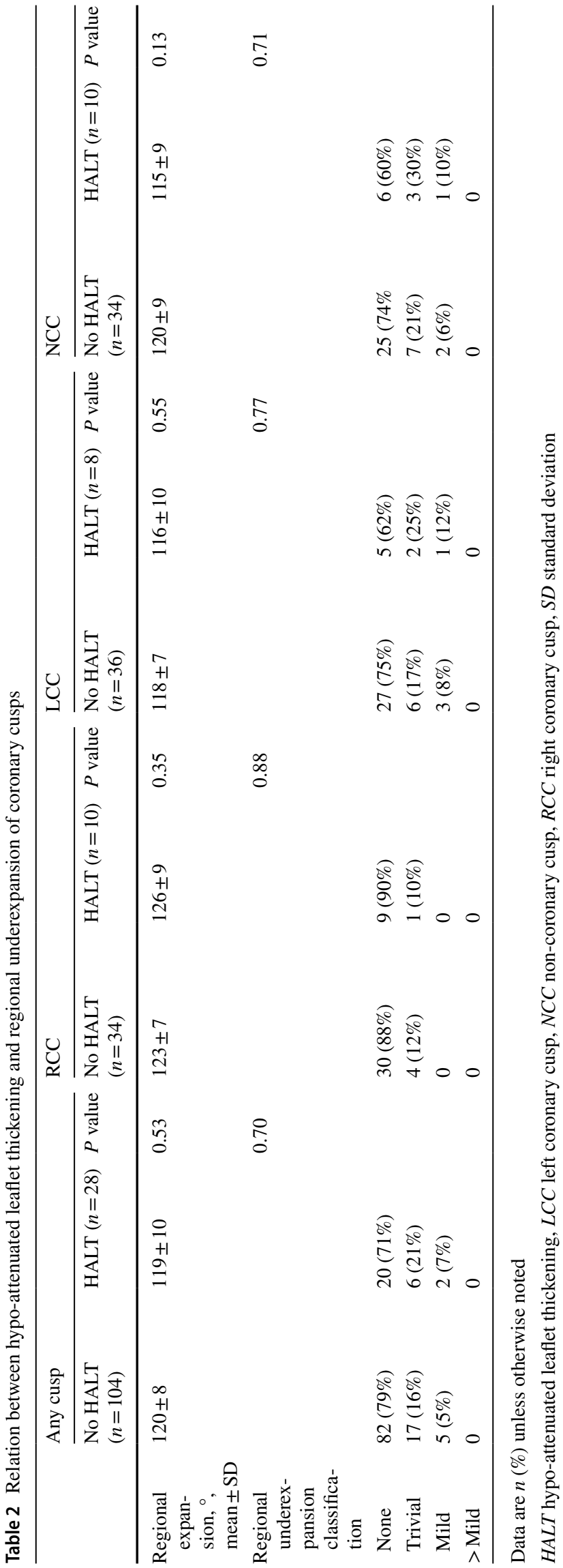




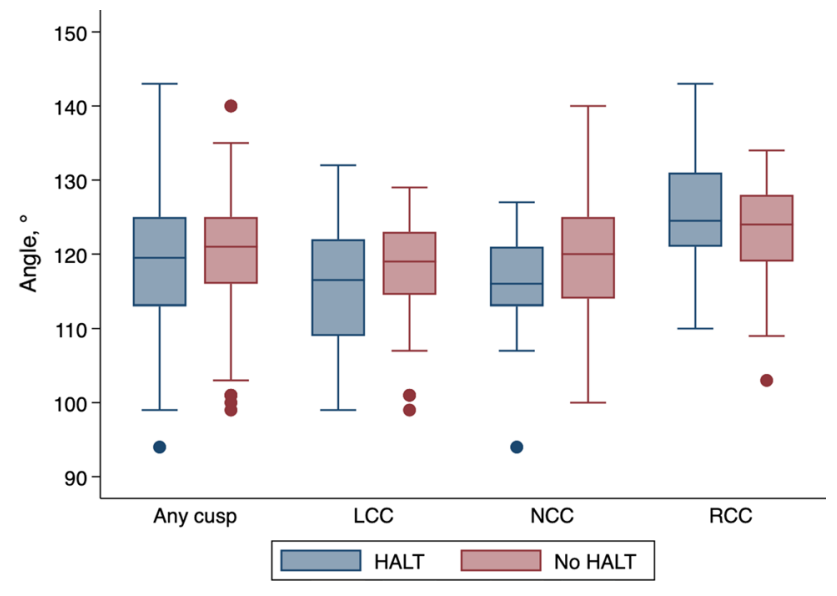

Fig. 5 Regional expansion, measured using the angle formed by the border of each prosthetic leaflet and the center point of the valve, in relation to hypo-attenuated leaflet thickening, in each coronary cusp. The whiskers denote the lowest/highest data points still within 1.5 interquartile range of the lower/upper quartile. The points denote outliers. $L C C$ left coronary cusp, NCC non-coronary cusp, $R C C$ right coronary cusp

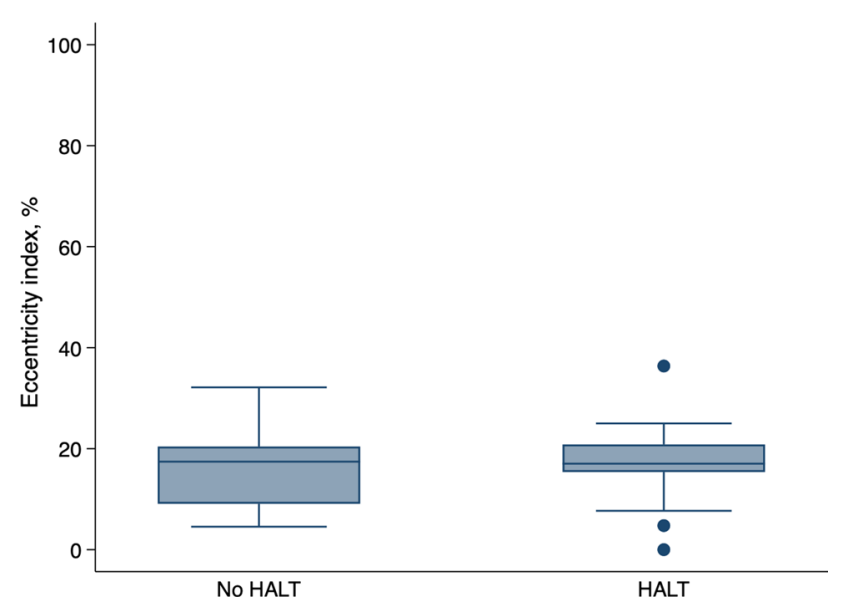

Fig. 6 The degree of eccentricity of the implanted prosthesis in relation to hypo-attenuated leaflet thickening. The whiskers denote the lowest/highest data points still within 1.5 interquartile range of the lower/upper quartile. The points denote outliers

\section{Discussion}

In this study, the prevalence of HALT and overall underexpansion was high but the prevalence of regional underexpansion low in the Perceval sutureless bioprosthetic valve. Patients with an overall expansion $<80 \%$ had a higher incidence of HALT though not significantly. To determine if severe oversizing increases the risk for HALT in this prosthesis would require further study. Regional underexpansion in the Perceval sutureless bioprosthetic valve was not associated with HALT.

HALT has been found to occur in both transcatheter and surgical bioprosthetic aortic valves. Studies have shown a varying prevalence ranging from $<5 \%$ to as high as $40 \%$ [1, 11]. In a large observational study of 931 patients, HALT occurred more commonly in TAVI patients compared to patients receiving surgical aortic valves (13\% vs. 4\%) [2]. In our study, the prevalence of HALT was high (39.1\%). An explanation could be that CT examinations in our study where performed after a median of 491 days which is relatively late compared to previous studies. Leaflet thrombosis has been speculated to be the underlying cause of HALT. The phenomenon is less prevalent in patients on anticoagulation therapy with warfarin [2] and the condition has been shown to be at least temporarily reversible when treated with warfarin or novel oral anticoagulants [12]. However, aggressive anticoagulation strategies can be associated with an increased risk of adverse events which have to be weighed against the clinical implications of HALT. In a recent trial, TAVI patients treated with Rivaroxaban had increased risks of all-cause mortality, thromboembolic events, and bleeding compared to those on antiplatelet therapy [13].

Recent findings suggest that HALT may affect clinical outcomes. A meta-analysis including 6 studies and a total 1704 patients showed that cerebrovascular events, defined as a composite of stroke or transient ischaemic attack, occurred significantly more frequently in patients with subclinical leaflet thrombosis [4]. Furthermore, it has been speculated that HALT may be related to reduced leaflet durability as prosthetic valve thrombosis is associated with prosthesis dysfunction and reduced durability [11, 14].

There is not sufficient research to explain why some valves or leaflets would be more prone to HALT than others. However, it has been suggested that underexpansion of the valve could be associated with leaflet anomalies. A study that included 75 patients who underwent TAVI with three different transcatheter valves showed an increased prevalence of HALT in patients with regional TAVI valve underexpansion, hypothesizing that an underexpanded "wrinkled" leaflet is more prone to thrombosis on its surface. Regional underexpansion was prevalent in 31-37\% of the leaflet sites, depending on the type of transcatheter valve [5]. Other possible causes of HALT such as trauma to the valve before or during implantation and inflammatory response related to the procedure remain to be determined [15]. In the surgically implanted bioprostheses in the present study, regional underexpansion was rare. When present, regional underexpansion was mild and was not associated with a higher prevalence of HALT. This might be explained by the removal of the native aortic valve and decalcification of the aortic annulus performed in surgical aortic valve replacement. This procedure likely limits 
the risk of asymmetrical valve expansion owing to uneven areal distribution of residual aortic valve and annular calcification.

In surgically implanted Perceval sutureless bioprostheses, overall underexpansion and failure to achieve circularity have been hypothesized to affect leaflet function [6]. One study showed that the degree of oversizing of the implanted prosthesis (calculated as the ratio between the native aortic annulus CSA and the in vitro CSA of the implanted prosthesis), and thereby overall underexpansion, was the most important predictor for increased postoperative transprosthetic gradients [7]. In the present study, there was no association between overall expansion or eccentricity of the bioprosthesis and the prevalence of HALT. In the TAVI population, an oversizing $>20 \%$ is rarely accepted and is considered severe [16]. In our study, only ten patients $(21.7 \%)$ reached an acceptable overall expansion of $\geq 80 \%$ of the prosthesis. HALT was less common in patients with overall expansion $\geq 80 \%$ compared to patients with $<80 \%$ overall expansion though not significantly. General oversizing of the Perceval sutureless prosthesis have been previously reported and suggestions to modify the sizing process to adjust for this [7] have been implemented.

This was a single-center observational study with the aim to investigate the potential relationship between overall and regional underexpansion and HALT in the Perceval sutureless bioprosthetic valve. It was not designed to find predictors of, nor adverse events associated with, HALT. Patients were not included in the study at the time of valve replacement and, therefore, the time intervals between AVR and 4D cardiac CT examinations varied considerably. However, very few previous studies have investigated the potential association between bioprosthetic valve geometry and HALT. The Perceval sutureless prosthesis has similarities with both conventional surgical as well as transcatheter aortic bioprostheses, making investigation of causes of HALT of this prosthesis of particular interest.

In conclusion, the prevalence of HALT and overall underexpansion was high in the Perceval sutureless bioprosthetic valve but overall underexpansion was not associated with HALT. Patients with an overall expansion $<80 \%$ had a higher incidence of HALT though not significantly. To determine if severe oversizing increases the risk for HALT in this prosthesis requires further study. Regional underexpansion in the Perceval sutureless bioprosthetic valve was not associated with HALT.

Acknowledgments Open access funding provided by Karolinska Institute.

Funding This study was supported by the Mats Kleberg Foundation and a donation from Mr. Fredrik Lundberg. Magnus Dalén was financially supported by a research Grant from Karolinska Institutet (Grant number: 2018-02009).

\section{Compliance with ethical standards}

Conflict of interest All authors declare that they have no conflict of interest.

Open Access This article is licensed under a Creative Commons Attribution 4.0 International License, which permits use, sharing, adaptation, distribution and reproduction in any medium or format, as long as you give appropriate credit to the original author(s) and the source, provide a link to the Creative Commons licence, and indicate if changes were made. The images or other third party material in this article are included in the article's Creative Commons licence, unless indicated otherwise in a credit line to the material. If material is not included in the article's Creative Commons licence and your intended use is not permitted by statutory regulation or exceeds the permitted use, you will need to obtain permission directly from the copyright holder. To view a copy of this licence, visit http://creativecommons.org/licenses/by/4.0/.

\section{References}

1. Makkar RR, Fontana G, Jilaihawi H, Chakravarty T, Kofoed KF, De Backer O, Asch FM, Ruiz CE, Olsen NT, Trento A, Friedman J, Berman D, Cheng W, Kashif M, Jelnin V, Kliger CA, Guo H, Pichard AD, Weissman NJ, Kapadia S, Manasse E, Bhatt DL, Leon MB, Søndergaard L (2015) Possible subclinical leaflet thrombosis in bioprosthetic aortic valves. N Engl J Med 373(21):2015-2024

2. Chakravarty T, Søndergaard L, Friedman J, De Backer O, Berman D, Kofoed KF, Jilaihawi H, Shiota T, Abramowitz Y, Jørgensen TH, Rami T, Israr S, Fontana G, de Knegt M, Fuchs A, Lyden P, Trento A, Bhatt DL, Leon MB, Makkar RR, RESOLVE; SAVORY Investigators (2017) Subclinical leaflet thrombosis in surgical and transcatheter bioprosthetic aortic valves: an observational study. Lancet 389(10087):2383-2392

3. Dalén M, Sartipy U, Cederlund K, Franco-Cereceda A, Svensson A, Themudo R, Svenarud P, Bacsovics Brolin E (2017) Hypoattenuated leaflet thickening and reduced leaflet motion in sutureless bioprosthetic aortic valves. J Am Heart Assoc 6(8):e005251

4. Rashid HN, Gooley RP, Nerlekar N, Ihdayhid AR, McCormick LM, Nasis A, Cameron JD, Brown AJ (2018) Bioprosthetic aortic valve leaflet thrombosis detected by multidetector computed tomography is associated with adverse cerebrovascular events: a meta-analysis of observational studies. EuroIntervention 13(15):e1748-e1755

5. Fuchs A, De Backer O, Brooks M, de Knegt MC, Bieliauskas G, Yamamoto M, Yanagisawa R, Hayashida K, Søndergaard L, Kofoed KF (2017) Subclinical leaflet thickening and stent frame geometry in self-expanding transcatheter heart valves. EuroIntervention 13(9):e1067-e1075

6. Cerillo AG, Amoretti F, Mariani M, Chiappino D (2018) Sutureless valve thrombosis: the role of stent geometry. Ann Thorac Surg 105(3):986-988

7. Cerillo AG, Amoretti F, Mariani M, Cigala E, Murzi M, Gasbarri T, Solinas M, Chiappino D (2018) Increased gradients after aortic valve replacement with the perceval valve: the role of oversizing. Ann Thorac Surg 106(1):121-128

8. Fischlein T, Meuris B, Hakim-Meibodi K, Misfeld M, Carrel T, Zembala M, Gaggianesi S, Madonna F, Laborde F, Asch F, Haverich A, CAVALIER Trial Investigators (2016) The sutureless 
aortic valve at 1 year: a large multicenter cohort study. J Thorac Cardiovasc Surg 151(6):1617-1626

9. Budoff MJ, Cohen MC, Garcia MJ, Hodgson JM, Hundley WG, Lima JA, Manning WJ, Pohost GM, Raggi PM, Rodgers GP, Rumberger JA, Taylor AJ, Creager MA, Hirshfeld JW Jr, Lorell BH, Merli G, Rodgers GP, Tracy CM, Weitz HH, American College of Cardiology Foundation; American Heart Association; American College of Physicians Task Force on Clinical Competence; American Society of Echocardiography; American Society of Nuclear Cardiology; Society of Atherosclerosis Imaging; Society for Cardiovascular Angiography \& Interventions; Society of Cardiovascular Computed Tomography (2005) ACCF/AHA clinical competence statement on cardiac imaging with computed tomography and magnetic resonance. Circulation 112(4):598-617

10. Pache G, Schoechlin S, Blanke P, Dorfs S, Jander N, Arepalli CD, Gick M, Buettner HJ, Leipsic J, Langer M, Neumann FJ, Ruile P (2016) Early hypo-attenuated leaflet thickening in balloon-expandable transcatheter aortic heart valves. Eur Heart J 37(28):2263-2271

11. Kanjanauthai S, Pirelli L, Nalluri N, Kliger CA (2018) Subclinical leaflet thrombosis following transcatheter aortic valve replacement. J Interv Cardiol 31(5):640-647

12. Ruile P, Jander N, Blanke P, Schoechlin S, Reinohl J, Gick M, Rothe J, Langer M, Leipsic J, Buettner HJ, Neumann FJ, Pache G (2017) Course of early subclinical leaflet thrombosis after transcatheter aortic valve implantation with or without oral anticoagulation. Clin Res Cardiol 106(2):85-95

13. Guedeney P, Mehran R, Collet JP, Claessen BE, Ten Berg J, Dangas GD (2019) Antithrombotic therapy after transcatheter aortic valve replacement. Circ Cardiovasc Interv 12(1):e007411

14. Roudaut R, Serri K, Lafitte S (2007) Thrombosis of prosthetic heart valves: diagnosis and therapeutic considerations. Heart 93(1):137-142

15. Amahzoune B, Bruneval P, Allam B, Lafont A, Fabiani JN, Zegdi R (2013) Traumatic leaflet injury during the use of percutaneous valves: a comparative study of balloon- and self-expandable valved stents. Eur J Cardiothorac Surg 43(3):488-493

16. Dvir D, Webb JG, Piazza N, Blanke P, Barbanti M, Bleiziffer S, Wood DA, Mylotte D, Wilson AB, Tan J, Stub D, Tamburino C, Lange R, Leipsic J (2015) Multicenter evaluation of transcatheter aortic valve replacement using either SAPIEN XT or CoreValve: degree of device oversizing by computed-tomography and clinical outcomes. Catheter Cardiovasc Interv 86(3):508-515

Publisher's Note Springer Nature remains neutral with regard to jurisdictional claims in published maps and institutional affiliations. 